\title{
Improved Results on Robust Stability of Multivariable Interval Control Systems ${ }^{1}$
}

\author{
Zhizhen Wang(1) $\quad$ Long Wang ${ }^{(2)} \quad$ Wensheng $\mathrm{Yu}^{(1)}$ \\ (1) Institute of Automation, Chinese Academy of Sciences, \\ Beijing, 100080, CHINA \\ (2) Center for Systems and Control, Department of Mechanics and Engineering Science, \\ Peking University, Beijing 100871, CHINA
}

\begin{abstract}
For interval polynomial matrices, we identify the minimal testing set, whose stability can guarantee that of the whole uncertain set. Our results improve the conclusions given by Kamal and Dahleh.
\end{abstract}

Keywords: Robust Stability, Polynomial Matrices, Interval Polynomials, Generalized Kharitonov's Theorem.

\section{Introduction}

A control system is said to be robust if it retains an assigned degree of stability or performance under perturbations. Robustness is considered as an elementary aspect in the analysis and design of control systems. There are several research lines for different models of uncertainties. Robust control under structured perturbations is an active area in recent years ${ }^{[1]-[11]}$.

The parametric robust stability problem was initiated by Kharitonov(1978) ${ }^{[1]}$, who gave a sufficient and necessary condition for the robust stability of interval polynomials. Chapellat et al.(1989) ${ }^{[6]}$ proved the Box Theorem for the multilinear model $P(s)=U(s) V(s)+X(s) Y(s)$.

When considering multivariable systems, the robust stability problem becomes more complicated $[3,5,6,7,8,9]$. Ref. [7] and Ref. [8] discussed the scalar matrix case and pointed out that a finite test does not hold. Ref. [5], [9] presented an $n$-dimensional result for polynomial matrices. Ref. [3] gave a $2 n$-dimensional result for the model $B(s) A(s)+D(s) C(s)$, where $B(s), D(s)$ are interval polynomial matrices and $A(s), C(s)$ are fixed ones.

The purpose of this paper is to improve the robust stability criteria for multivariable interval control systems obtained by Kamal and Dehleh. By exploiting the uncertainty structures, we are able to reduce the computational burden in checking robust stability.

\section{Preliminaries}

Definition 2.1 Given an interval polynomial set $r(s)=\sum_{i=0}^{m} r_{i} s^{i}, \quad r_{i} \in\left[r_{i}^{L}, r_{i}^{U}\right]$, its Kharitonov vertex set is $r_{V}(s)=\left\{r_{1}(s), r_{2}(s), r_{3}(s), r_{4}(s)\right\}$, and its Kharitonov edge set is $r_{E}(s)=\left\{\lambda r_{i}(s)+(1-\right.$

\footnotetext{
${ }^{1}$ Supported by National Key Project and National Natural Science Foundation of China(69925307).
} 
$\left.\lambda) r_{j}(s), \quad(i, j) \in\{(1,2),(2,4),(4,3),(3,1)\} \quad \lambda \in[0,1]\right\}$, where

$$
\begin{aligned}
& r_{1}(s)=r_{0}^{L}+r_{1}^{L} s+r_{2}^{U} s^{2}+r_{3}^{U} s^{3}+r_{4}^{L} s^{4}+\ldots \\
& r_{2}(s)=r_{0}^{L}+r_{1}^{U} s+r_{2}^{U} s^{2}+r_{3}^{L} s^{3}+r_{4}^{L} s^{4}+\ldots \\
& r_{3}(s)=r_{0}^{U}+r_{1}^{L} s+r_{2}^{L} s^{2}+r_{3}^{U} s^{3}+r_{4}^{U} s^{4}+\ldots \\
& r_{4}(s)=r_{0}^{U}+r_{1}^{U} s+r_{2}^{L} s^{2}+r_{3}^{L} s^{3}+r_{4}^{U} s^{4}+\ldots
\end{aligned}
$$

Definition 2.2 A polynomial is stable if all its zeroes belong to the open left half plane. A polynomial matrix is stable if its determinant is stable.

Lemma 1 (Box Theorem ${ }^{[6]}$ ) Consider the polynomial family $\Delta(s)=\left\{\delta(s, p)=F_{1}(s) P_{1}(s)+\right.$ $\ldots+F_{m}(s) P_{m}(s), P_{i}(s)$ are interval polynomials, $F_{i}(s)$ are fixed polynomials, $\left.i=1, \ldots, m\right\}$ and suppose $\Delta(s)$ is without degree dropping. Then $\Delta(s)$ is Hurwitz stable if and only if $\Delta_{E}(s)$ is Hurwitz stable, where $\Delta_{E}(s)=\cup_{l=1}^{m}\left\{\sum_{i=1}^{l-1} F_{i}(s) K_{P_{i}}(s)+F_{l}(s) E_{P_{l}}(s)+\sum_{i=l+1}^{m} F_{i}(s) K_{P_{i}}(s)\right\}$ and $K_{P_{i}}(s), E_{P_{i}}(s)$ are the Kharitonov vertex set, Kharitonov edge set of $P_{i}(s)$, respectively. (Here we take $\sum_{i=r}^{t} f_{i}=0$ if $r>t)$.

Let $A(s)=\left(a_{i j}(s)\right)_{n \times n}, C(s)=\left(c_{i j}(s)\right)_{n \times n}$ be two fixed matrices, where $A(s)=\left(a_{i j}(s)\right)_{n \times n}$ means that the matrix is an $n \times n$ one and its $i j$-th entry is $a_{i j}(s)$. Given two interval polynomial matrices $B(s)=\left(b_{i j}(s)\right)_{n \times n}, D(s)=\left(d_{i j}(s)\right)_{n \times n}$, i.e., $b_{i j}(s), d_{i j}(s)$ are interval polynomials. Let

$$
\begin{array}{r}
B(i j)=\left\{b_{i j}(s): b_{i j}(s)=b_{i j}^{(0)}+b_{i j}^{(1)} s+\ldots+b_{i j}^{(n)} s^{n}, b_{i j}^{(k)} \in\left[\underline{b}_{i j}^{(k)}, \bar{b}_{i j}^{(k)}\right]\right\} \\
D(i j)=\left\{d_{i j}(s): d_{i j}(s)=d_{i j}^{(0)}+d_{i j}^{(1)} s+\ldots+d_{i j}^{(n)} s^{n}, d_{i j}^{(k)} \in\left[\underline{d}_{i j}^{(k)}, \bar{d}_{i j}^{(k)}\right]\right\} \\
\mathcal{B}(s)=\left\{B(s): B(s)=\left(b_{i j}(s)\right)_{n \times n}, b_{i j}(s) \in B(i j)\right\} \\
\mathcal{D}(s)=\left\{D(s): D(s)=\left(d_{i j}(s)\right)_{n \times n}, d_{i j}(s) \in D(i j)\right\}
\end{array}
$$

Denote Kharitonov vertex sets and Kharitonov edge sets of $B(i j), D(i j)$ as $\mathcal{B}_{V}(i j), \mathcal{D}_{V}(i j), \mathcal{B}_{E}(i j)$ and $\mathcal{D}_{E}(i j)$ respectively.

\section{Main Results}

In this part, we consider the uncertain family

$$
\mathcal{M}(s)=\mathcal{B}(s) A(s)+\mathcal{D}(s) C(s)
$$

Let $S_{n}$ denote the set of all bijections of the set $\{1, \ldots, n\}$ onto itself. The following theorem was given by Kamal et al.(1996).

Proposition $1^{[9]} \mathcal{M}(s)$ is stable for all $B(s) \in \mathcal{B}(s), D(s) \in \mathcal{D}(s)$ if and only if $\mathcal{M}(s)$ is stable for all $B(s) \in \mathcal{B}_{E}(s), D(s) \in \mathcal{D}_{E}(s)$, where

$$
\begin{aligned}
& \mathcal{B}_{E}(s)=\left\{\begin{array}{ll} 
& b_{i j}(s) \in \mathcal{B}_{E}(i j), j=\sigma(i) \\
B(s) \in \mathcal{B}(s): & b_{i j}(s) \in \mathcal{B}_{V}(i j), j \neq \sigma(i) \\
& \sigma \in S_{n}
\end{array}\right\} \\
& \mathcal{D}_{E}(s)=\left\{\begin{array}{ll} 
& d_{i j}(s) \in \mathcal{D}_{E}(i j), j=\sigma(i) \\
D(s) \in \mathcal{D}(s): \quad & d_{i j}(s) \in \mathcal{D}_{V}(i j), j \neq \sigma(i) \\
\sigma \in S_{n}
\end{array}\right\}
\end{aligned}
$$

As a generalization of the Generalized Kharitonov Theorem, Proposition 1 addresses the robust stability of $\mathcal{M}(s)$ and reduce it to $4^{2 n^{2}}(n !)^{2}$ robust stability problems involving determinants with $2 n$ parameters. In this paper, we will show that, by making use of the uncertainty structure information, the original robust stability problem can be reduced to $\sum_{t=0}^{n}\left(C_{n}^{t}\right)^{2} 4^{2 n^{2}}(n !)$ robust stability problems 
involving $n$ parameters, where $C_{n}^{t}=\frac{n !}{t !(n-t) !}$. In the sequel, the notation $X \times Y$ stands for the Cartesian product, which is the set of all ordered pairs $(x, y)$, where $x \in X, y \in Y$. The following lemma is needed before we present our main result.

Lemma $2 A(s), B(s), C(s)$ and $D(s)$ are defined as before. Let

$$
\begin{array}{r}
N^{(i)}(s)=\left\{\begin{array}{r}
\left.\exists(s) A(s)+D(s) C(s): \begin{array}{l}
\exists k_{1}, k_{2} \in\{1, \ldots, n\} ; l=1, \ldots, n \\
b_{i k_{1}} \in \mathcal{B}_{E}\left(i k_{1}\right) ; b_{i l} \in \mathcal{B}_{V}(i l), l \neq k_{1} \\
d_{i k_{1}} \in \mathcal{D}_{E}\left(i k_{2}\right) ; d_{i l} \in \mathcal{D}_{V}(i l), l \neq k_{2}
\end{array}\right\} \\
\quad \exists k \in\{1, \ldots, n\} ; l=1, \ldots, n \\
N_{E}^{(i)}(s)=\left\{B(s) A(s)+D(s) C(s): \begin{array}{l}
b_{i k} \times d_{i k} \in\left(\mathcal{B}_{E}(i k) \times \mathcal{D}_{V}(i k)\right) \cup\left(\mathcal{B}_{V}(i k) \times \mathcal{D}_{E}(i k)\right) \\
b_{i l} \times d_{i l} \in\left(\mathcal{B}_{V}(i l) \times \mathcal{D}_{V}(i l)\right), l \neq k
\end{array}\right\}
\end{array}\right.
\end{array}
$$

Then, for all $i \in\{1, \ldots, n\}, N^{(i)}(s)$ is stable if and only if $N_{E}^{(i)}(s)$ is stable .

Proof (Sufficiency) Suppose that $N_{E}^{(i)}(s)$ is stable, our aim is to prove that $N^{(i)}(s)$ is stable. For any $N(s) \in N^{(i)}(s)$, there exist $k_{1}, k_{2} \in\{1, \ldots, n\}$ such that

$$
\begin{array}{ll}
b_{i k_{1}} \in \mathcal{B}_{E}\left(i k_{1}\right) ; & b_{i l} \in \mathcal{B}_{V}(i l), l \neq k_{1}, l=1, \ldots, n \\
d_{i k_{1}} \in \mathcal{D}_{E}\left(i k_{2}\right) ; & d_{i l} \in \mathcal{D}_{V}(i l), l \neq k_{2}, l=1, \ldots, n
\end{array}
$$

and

$$
N(s)=\left(\begin{array}{lll}
\sum_{k}\left(b_{1 k}(s) a_{k 1}(s)+d_{1 k}(s) c_{k 1}(s)\right) & \ldots & \sum_{k}\left(b_{1 k}(s) a_{k n}(s)+d_{1 k}(s) c_{k n}(s)\right) \\
\ldots & \ldots & \ldots \\
\sum_{k}\left(b_{i k}(s) a_{k 1}(s)+d_{i k}(s) c_{k 1}(s)\right) & \ldots & \sum_{k}\left(b_{i k}(s) a_{k n}(s)+d_{i k}(s) c_{k n}(s)\right) \\
\ldots & \ldots & \ldots \\
\sum_{k}\left(b_{n k}(s) a_{k 1}(s)+d_{n k}(s) c_{k 1}(s)\right) & \ldots & \sum_{k}\left(b_{n k}(s) a_{k n}(s)+d_{n k}(s) c_{k n}(s)\right)
\end{array}\right)
$$

By definition, $N(s)$ is stable if and only if $\operatorname{det} N(s)$ is stable. By Laplace formula, expanding the determinant along the $i$-th row, we have

$$
\operatorname{det} N(s)=\sum_{k}\left(b_{i k}(s) a_{k 1}(s)+d_{i k}(s) c_{k 1}(s)\right) N_{i 1}+\ldots+\sum_{k}\left(b_{i k}(s) a_{k n}(s)+d_{i k}(s) c_{k n}(s)\right) N_{i n},
$$

where $N_{i j}$ is the algebraic complementary minor of the $i j$-th entry of $N(s)$, which is independent of $b_{i 1}(s), \ldots, b_{i n}(s), d_{i 1}(s), \ldots, d_{i n}(s)$. By a simple manipulation, we have

$$
\begin{aligned}
\operatorname{det} N(s) & =\sum_{j=1}^{n} b_{i j}(s)\left(\sum_{k=1}^{n} a_{j k}(s) N_{i k}\right)+\sum_{j=1}^{n} d_{i j}(s)\left(\sum_{k=1}^{n} c_{j k}(s) N_{i k}\right) \\
& =b_{i k_{1}}(s) \delta_{1}(s)+d_{i k_{2}}(s) \delta_{2}(s)+\delta_{3}(s)
\end{aligned}
$$

where $\delta_{l}(s)$ is a term which is independent of $b_{i 1}(s), \ldots, b_{i n}(s), d_{i 1}(s), \ldots, d_{i n}(s)$. Denote

$$
\left\{\begin{array}{l}
b_{i k_{1}}(s) \times d_{i k_{2}}(s) \in\left(\mathcal{B}_{E}\left(i k_{1}\right) \times \mathcal{D}_{V}\left(i k_{2}\right)\right) \cup\left(\mathcal{B}_{V}\left(i k_{1}\right) \times \mathcal{D}_{E}\left(i k_{2}\right)\right) \\
b_{i l}(s) \in \mathcal{B}_{V}(i l), l \neq k_{1}, l=1, \ldots, n \\
d_{i l}(s) \in \mathcal{D}_{V}(i l), l \neq k_{2}, l=1, \ldots, n
\end{array}\right.
$$

By Lemma 1, $\operatorname{det} N(s)$ is stable for $N(s)$ satisfying $(10),(11)$ if and only if $\operatorname{det} N(s)$ is stable for $N(s)$ satisfying (11),(12). Since $N_{E}^{(i)}(s)$ is stable, it shows that $N(s)$ is stable. Hence, sufficiency is proved. (Necessary) This part is obvious, since $N_{E}^{(i)}(s) \subset N^{(i)}(s)$.

Before presenting our main result, we first define some notations. Let $T_{n}$ be the set of all functions from the set $\{1, \ldots, n\}$ into itself. Take

$$
\mathcal{B D}=\left\{\begin{array}{lll} 
& b_{i j}(s) \times d_{i j}(s) \in\left(\mathcal{B}_{E}(i j) \times \mathcal{D}_{V}(i j)\right) \cup\left(\mathcal{B}_{V}(i j) \times \mathcal{D}_{E}(i j)\right) & j=\eta(i) \\
B(s) \times D(s): & b_{i j}(s) \times d_{i j}(s) \in \mathcal{B}_{V}(i j) \times \mathcal{D}_{V}(i j) & j \neq \eta(i) \\
& \eta \in T_{n}
\end{array}\right\}
$$


and

$$
\mathcal{B}_{E} \mathcal{D}_{E}=\left\{B(s) \times D(s): B(s) \times D(s) \in \mathcal{B}_{E}(s) \times \mathcal{D}_{E}(s)\right\} .
$$

By Proposition 1 and Lemma 2, we get the main result of this paper.

Theorem $1 \mathcal{M}(s)$ is stable for all $B(s) \in \mathcal{B}(s), D(s) \in \mathcal{D}(s)$ if and only if $\mathcal{M}(s)$ is stable for all $B(s) \times D(s) \in \mathcal{B D} \cap \mathcal{B}_{E} \mathcal{D}_{E}$.

Proof By Proposition 1, $\mathcal{M}(s)$ is stable for all $B(s) \in \mathcal{B}(s), D(s) \in \mathcal{D}(s)$ if and only if $\mathcal{M}(s)$ is stable for all $B(s) \times D(s) \in \mathcal{B}_{E} \mathcal{D}_{E}$. For any $n(s) \in \mathcal{M}(s)$ with $B(s) \times D(s) \in \mathcal{B}_{E} \mathcal{D}_{E}$, fixing the rows from the second to the $n$-th and applying Lemma 2 to the first row, we have

$$
\begin{gathered}
\mathcal{M}(s) \text { is stable for all } B(s) \in \mathcal{B}(s), D(s) \in \mathcal{D}(s) \text { if and only if } \\
\mathcal{M}(s) \text { is stable for all } B(s) \times D(s) \in \mathcal{B D}_{1} \cap \mathcal{B}_{E} \mathcal{D}_{E}
\end{gathered}
$$

where $\mathcal{B D}_{1} \subset \mathcal{B}(s) \times \mathcal{D}(s)$ satisfies (12) when $i=1$. Repeating the same procedure for the remaining rows consecutively, we get the conclusions.

Remark 1 Theorem 1 shows that, the criterion for the robust stability of interval polynomial matrices can be greatly simplified. The dimension of the minimal testing set is reduced to $n$.

Remark 2 Theorem 1 establishes the minimal dimensional testing criteria for robust stability of multivariable control systems. By making use of the uncertainty information of $\mathcal{B}(s)$ and $\mathcal{D}(s)$, i.e., not only making use of the uncertainty information in $\mathcal{B}(s)$ and $\mathcal{D}(s)$ separately (this is exactly what Kamal and Dehleh did), but also making use of the uncertainty information collectively, we are able to improve the robust stability results given by Kamal and Dehleh.

Remark 3 Theorem 1 allows us to improve the main results of Kamal and Dehleh.

\section{Applications}

Based on Proposition 1, Kamal et al.(1996) address several robustness problems as follows.

Proposition 2([3]) Let $P(S)=B(S) D^{-1}(s)$ and $K(s)=A(s) C^{-1}(s)$ be right coprime factorization and left coprime factorization of $P(s)$ and $K(s)$ respectively over the ring of polynomial matrices. Let $B(s) \in \mathcal{B}(s)$ and $D(s) \in \mathcal{D}(s)$, and let $K(s)$ be fixed. Then the closed loop system in Fig. 1 is stable for all $B(s) \in \mathcal{B}(s), D(s) \in \mathcal{D}(s)$ if and only if it is stable for all $B(s) \in \mathcal{B}_{E}(s), D(s) \in \mathcal{D}_{E}(s)$.

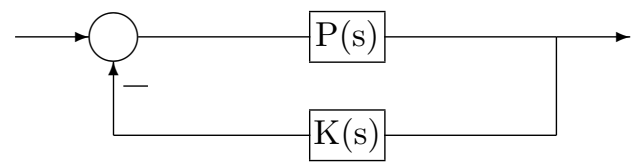

Fig 1: Feedback Systems

Let $H_{\infty}^{n \times m}\left(\mathcal{C}_{+}\right)$be the space of matrix valued functions $F(s)$ that are analytic in $\mathcal{C}_{+}$and bounded on the $j \omega$-axis with the norm $\|F\|_{\infty}=\sup _{\omega \in \mathcal{R}} \sigma_{\max }(F(j \omega))$.

Proposition $3\left(^{[3]}\right)$ Let $\mathcal{G}(s) \subset H_{\infty}^{n \times n}\left(\mathcal{C}_{+}\right)$be a family of proper interval transfer function matrices. Let $G(s)=B(s) D^{-1}(s)$ be a right coprime description of $G(s)$ over the ring of polynomial matrices, with $D(s)$ column reduced; then

1) $\|G\|_{\infty}<1$ for all $B(s) \in \mathcal{B}(s), D(s) \in \mathcal{D}(s)$ if and only if $\|G\|_{\infty}<1$ for all $B(s) \in \mathcal{B}_{E}(s), D(s) \in \mathcal{D}_{E}(s)$. 2) $G(s)$ is strictly positive real for all $B(s) \in \mathcal{B}(s), D(s) \in \mathcal{D}(s)$ if and only if $G(s)$ is strictly positive real for all $B(s) \in \mathcal{B}_{E}(s), D(s) \in \mathcal{D}_{E}(s)$. 
A memoryless nonlinearity $\Delta_{s}:[0, \infty) \times \mathcal{R}^{n} \rightarrow \mathcal{R}^{n}$ is said to satisfy a sector condition if for all $y \in \mathcal{R}^{n}$ and $t \geq 0$,

$$
\left[\Delta_{s}(t, y)-K_{1} y\right]^{T}\left[\Delta_{s}(t, y)-K_{2} y\right] \geq 0
$$

for some real matrices $K_{1}$ and $K_{2}$, where $K=K_{2}-K_{1}$ is a symmetric positive definition matrix.

Proposition $\left.4{ }^{[3]}\right) G(s)=B(s) D^{-1}(s)$ is a family of interval proper stable systems, where $B(s) \in$ $\mathcal{B}(s), D(s) \in \mathcal{D}(s)$, and $\Delta_{s}$ is a set of memoryless nonlinearities that satisfy the sector condition globally with $K>0$, then the system is globally stable if there is $\eta \geq 0$ such that $G(\infty)=0$ and

$$
Z(j \omega)=I+(1+j \omega \eta) K G(j \omega)>0, \quad \forall \omega \in \mathcal{R}
$$

for all $B(s) \in \mathcal{B}_{E}(s), D(s) \in \mathcal{D}_{E}(s)$.

By resort to Theorem 1, all testing sets above can be reduced to lower dimensional ones.

Theorem 2 Under the same assumptions as in Proposition 2, the closed loop system in Fig. 1 is stable for all $B(s) \in \mathcal{B}(s), D(s) \in \mathcal{D}(s)$ if and only if it is stable for all $B(s) \times D(s) \in \mathcal{B D} \cap \mathcal{B}_{E} \mathcal{D}_{E}$.

Theorem 3 Under the same assumptions as in Proposition 3, we have

1) $\|G\|_{\infty}<1$ for all $B(s) \in \mathcal{B}(s), D(s) \in \mathcal{D}(s)$ if and only if $\|G\|_{\infty}<1$ for all $B(s) \times D(s) \in \mathcal{B D} \cap \mathcal{B}_{E} \mathcal{D}_{E}$.

2) $G(s)$ is strictly positive real for all $B(s) \in \mathcal{B}(s), D(s) \in \mathcal{D}(s)$ if and only if $G(s)$ is strictly positive real for all $B(s) \times D(s) \in \mathcal{B D} \cap \mathcal{B}_{E} \mathcal{D}_{E}$.

Theorem 4 Under the same assumptions as in Proposition 4, the system is globally stable if there is $\eta \geq 0$ such that $G(\infty)=0$ and

$$
Z(j \omega)=I+(1+j \omega \eta) K G(j \omega)>0, \quad \forall \omega \in \mathcal{R}
$$

for all $B(s) \times D(s) \in \mathcal{B D} \cap \mathcal{B}_{E} \mathcal{D}_{E}$.

\section{Example}

Recall the two-link planar manipulator considered by Kamal and Dehleh. The characteristic polynomial of the closed loop system is

$$
T(\epsilon)=\operatorname{det}\left(M\left(\theta_{d}\right) s^{3}+K_{d} s^{2}+K_{p} s+K_{r}\right)
$$

where $\theta_{d} \in[0, \pi / 2]$ is the joint angle and

$$
\begin{aligned}
& M\left(\theta_{d}\right)=\left(\begin{array}{cc}
3+2 \cos \left(\theta_{d}\right) & 1+\cos \left(\theta_{d}\right) \\
1+\cos \left(\theta_{d}\right) & 1
\end{array}\right) \\
& K_{d}=\left(\begin{array}{cc}
k_{d 11} & k_{d 12} \\
k_{d 21} & k_{d 22} \\
k_{p 11} & k_{p 12} \\
k_{p 21} & k_{p 22}
\end{array}\right) \\
& K_{p}=\left(\begin{array}{ll}
k_{r 11} & k_{r 12} \\
k_{r 21} & k_{r 22}
\end{array}\right)
\end{aligned}
$$




$$
\begin{aligned}
& k_{d 11} \in[6.07-6.07 \epsilon, 6.07+6.07 \epsilon] \\
& k_{d 12} \in[2.22-2.22 \epsilon, 2.22+2.22 \epsilon] \\
& k_{d 21} \in[2.22-2.22 \epsilon, 2.22+2.22 \epsilon] \\
& k_{d 22} \in[1.62-1.62 \epsilon, 1.62+1.62 \epsilon] \\
& k_{p 11} \in[6.12-6.12 \epsilon, 6.12+6.12 \epsilon] \\
& k_{p 12} \in[2.24-2.24 \epsilon, 2.24+2.24 \epsilon] \\
& k_{p 21} \in[2.24-2.24 \epsilon, 2.24+2.24 \epsilon] \\
& k_{p 22} \in[1.64-1.64 \epsilon, 1.64+1.64 \epsilon] \\
& k_{r 11} \in[5.11-5.11 \epsilon, 5.11+5.11 \epsilon] \\
& k_{r 12} \in[1.87-1.87 \epsilon, 1.87+1.87 \epsilon] \\
& k_{r 21} \in[1.87-1.87 \epsilon, 1.87+1.87 \epsilon] \\
& k_{r 22} \in[1.37-1.37 \epsilon, 1.37+1.37 \epsilon] .
\end{aligned}
$$

Since for any fixed $\theta_{d} \in[0, \pi / 2]$, the inertia matrix

$$
M\left(\theta_{d}\right)=\left(\begin{array}{cc}
1 & \left(1+\cos \left(\theta_{d}\right)\right) \\
\left(-1+\cos \left(\theta_{d}\right)\right) & 1
\end{array}\right)\left(\begin{array}{ll}
1 & 0 \\
2 & 1
\end{array}\right)
$$

In what follows, we take the uncertain inertia matrix as

$$
M(s)=B(s) A(s)
$$

where $A(s)=\left(\begin{array}{ll}1 & 0 \\ 2 & 1\end{array}\right), B(s) \in \mathcal{B}(s)=\left\{\left(b_{i j}(s)\right)_{2 \times 2}: b_{i j}(s) \in B(i j)\right\}$ and

$$
\begin{array}{ll}
B(11)=s^{3} & B(12)=b_{12} \\
B(21)=b_{21} s^{3}, b_{21} \in[-1,0] & B(22)=s^{3}
\end{array}
$$

Let $\mathcal{D}(s)=K_{d} s^{3}+K_{p} s^{2}+K_{r}$, then

$$
\mathcal{D}(s)=\left\{\left(d_{i j}(s)\right): d_{i j}(s) \in D(i j)\right\},
$$

where $D(i j)=\left\{d_{i j}(s)=k_{d i j} s^{2}+k_{p i j} s+k_{r i j}: k_{d i j}, k_{p i j}\right.$ and $k_{r i j}$ are given in (15) $\}$. Thus, the characteristic polynomial of the uncertain system is

$$
T(\epsilon)=\operatorname{det}(B(s) A(s)+D(s)), \quad B(s) \in \mathcal{B}(s), D(s) \in \mathcal{D}(s)
$$

where $A(s)$ is a fixed matrix and $B(s), D(s)$ are interval polynomial matrices.

The Kharitonov polynomial vertex sets associated with $B(i j)$ and $D(i j)$ are as follows.

$$
\begin{gathered}
\mathcal{B}_{V}(11)=\left\{s^{3}\right\}=B(11), \quad \mathcal{B}_{V}(12)=\left\{s^{3}, 2 s^{3}\right\}, \quad \mathcal{B}_{V}(21)=\left\{-s^{3}, 0\right\}, \quad \mathcal{B}_{V}(22)=\left\{s^{3}\right\}=B(22), \\
\mathcal{D}_{V}(i j)=\left\{\begin{array}{ll}
k_{i j}^{(1)}=K_{d i j}^{U} s^{2}+K_{p i j}^{L} s+K_{r i j}^{L}, & k_{i j}^{(2)}=K_{d i j}^{U} s^{2}+K_{p i j}^{U} s+K_{r i j}^{L}, \\
k_{i j}^{(3)}=K_{d i j}^{L} s^{2}+K_{p i j}^{L} s+K_{r i j}^{U}, & k_{i j}^{(4)}=K_{d i j}^{L} s^{2}+K_{p i j}^{U} s+K_{r i j}^{U},
\end{array}\right\}
\end{gathered}
$$

where the upper bound and the lower bound of $K_{d i j}$ are denoted as $K_{d i j}^{U}, K_{d i j}^{L}$. And the Kharitonov edge sets associated with $B(i j)$ and $D(i j)$ are as follows.

$$
\begin{gathered}
\mathcal{B}_{E}(11)=\left\{s^{3}\right\}=B(11), \quad \mathcal{B}_{E}(12)=\left\{\lambda s^{3}+(1-\lambda) \cdot 2 s^{3}: \lambda \in[0,1]\right\}, \\
\mathcal{B}_{E}(21)=\left\{-\lambda s^{3}: \lambda \in[0,1]\right\}, \\
\mathcal{D}_{E}(i j)=\left\{\begin{array}{l}
\lambda k_{i j}^{(1)}+(1-\lambda) k_{i j}^{(2)}, \\
\lambda k_{i j}^{(2)}+(1-\lambda) k_{i j}^{(4)}, \\
\lambda k_{i j}^{(4)}+(1-\lambda) k_{i j}^{(3)}, \\
\lambda k_{i j}^{(3)}+(1-\lambda) k_{i j}^{(1)} .
\end{array}\right\}
\end{gathered}
$$


By Proposition 1 given by Kamal and Dehleh, for any $\epsilon, T(\epsilon)$ is robustly stable if and only if $T(\epsilon)$ is stable for all $B(s) \times D(s) \in \mathcal{B}_{E}(s) \times \mathcal{D}_{E}(s)$, where

$$
U_{1}=\left(\begin{array}{cc}
B(11) & B_{E}(12) \times \mathcal{D}_{E}(s)=U_{1} \cup U_{2} \\
B_{E}(21) & B(22) \\
B(11) & B_{E}(12) \\
B_{E}(21) & B(22)
\end{array}\right) \times\left(\begin{array}{cc}
D_{V}(11) & D_{E}(12) \\
D_{E}(21) & D_{V}(22) \\
D_{E}(11) & D_{V}(12) \\
D_{V}(21) & D_{E}(22)
\end{array}\right) .
$$

So, the robust stability of $T(\epsilon)$ is reduced to $2 \cdot 4^{6}$ robust stability problems involving determinants with four parameters. In the case, the testing set is a four-dimensional set. In the sequel, we apply Theorem 1 to simplify this problem further. That is to say, $T(\epsilon)$ is robustly stable if and only if $T(\epsilon)$ is stable for all $B(s) \times D(s) \in(\mathcal{B D}) \cap \mathcal{B}_{E} \mathcal{D}_{E}$, where

$$
\begin{array}{r}
(\mathcal{B D}) \cap \mathcal{B}_{E} \mathcal{D}_{E}=V_{1} \cup V_{2} \cup V_{3} \cup V_{4} \cup V_{5} \cup V_{6} \cup V_{7} \\
V_{1}=\left(\begin{array}{cc}
B(11) & B_{E}(12) \\
B_{E}(21) & B(22) \\
B(11) & B_{E}(12) \\
B_{V}(21) & B(22) \\
B(11) & B_{E}(12) \\
B_{V}(21) & B(22) \\
B(11) & B_{V}(12) \\
V_{3} & = \\
D_{E}(21) & B(22) \\
B(11) & B_{V}(12) \\
D_{E}(21) & B(22) \\
D_{V}(21) & D_{V}(22) \\
D_{E}(11) & D_{V}(12) \\
D_{V}(21) & D_{V}(22) \\
D_{V}(11) & D_{V}(12) \\
D_{E}(21) & D_{V}(22) \\
D_{V}(11) & D_{E}(12) \\
D_{V}(21) & D_{V}(22)
\end{array}\right) \times\left(\begin{array}{cc}
D_{V}(11) & D_{V}(12) \\
D_{V}(21) & D_{E}(22) \\
D_{E}(11) & D_{V}(12) \\
D_{V}(21) & D_{E}(22) \\
D_{V}(11) & D_{E}(12) \\
D_{E}(21) & D_{V}(22)
\end{array}\right) \\
V_{5}= \\
V_{6}=\left(\begin{array}{cc}
B_{V}(21) & B(22) \\
B(11) & B_{V}(12) \\
B_{V}(21) & B(22)
\end{array}\right) \times
\end{array}
$$

Clearly, the robust stability of $T(\epsilon)$ is reduced to $7 \cdot 4^{6}$ robust stability problems involving determinants with two parameters. In this case, the minimal testing set is two-dimensional.

Remark 4: A test with a parameter could involve infinite tests without parameter, such as an edge means infinite points. The significance of our result is that it is the minimal dimension test for such problem as (5). Furthermore, we consider the uncertainty in inertia matrix $M\left(\theta_{d}\right)$ in Example, which did not take into consideration in Ref. [3].

\section{Conclusions}

In this paper, interval transfer function matrices are studied. For a family of multivariable control systems having interval transfer functions matrices, a lower dimensional robust stability criterion has been established. This result improves the previous criterion obtained by Kamal and Dahleh, and can be used to establish improved lower dimensional robust performance criteria as well.

\section{References}

[1] V. L. Kharitonov. Asymptotic stability of an equilibrium position of a family of systems of linear differential equations, Differential'nye Uravneniya, vol.14, 2086-2088, 1978.

[2] A. C. Bartlett, C. V. Hollot and L. Huang. Root locations of an entire polytope of polynomials: It suffices to check the edges, Mathematics of Control, Signals, and Systems, vol.1, 61-71, 1988.

[3] F. Kamal and M. Dahleh. Robust stability of multivariable interval control systems, Int. J. Control, vol.64, 807-828, 1996. 
[4] L. Wang and L. Huang. Vertex results for uncertain systems, Int. J. Systems Science, vol.25, 541-549, 1994.

[5] L. Wang, Z. Z. Wang and W. S. Yu. Stability of polytopic polynomial matrices. Proc. American Conrol Conference, Arlington, Virginia, June: 4695-4696,2001.

[6] H. Chapellat and S. P. Bhattacharyya. A generalization of Kharitonov's theorem: robust stability of interval plants. IEEE Trans. on Automatic Control, vol.34, 306-311, 1989.

[7] S. Bialas. A necessary and sufficient condition for the stability of interval matrices, Int. J. Control, vol.37, 717-722, 1983.

[8] B. R. Barmish and C. V. Hollot. Counter-example to a recent result on the stability of interval matrices by S. Bialas, Int. J. Control, vol.39, 1103-1104, 1984.

[9] H. Kokame and T. Mori. A Kharitonov-like theorem for interval polynomial matrices, Systems and Control Letters, vol.16, 107-116, 1991.

[10] J. Ackermann, Robust Control: Systems with Uncertain Physical Parameters. Springer-Verlag, London, 1993.

[11] B. R. Barmish, New tools for robustness analysis, Proc. of IEEE Conf. on Decision and Control, 1-6, 1988. 